PROCEEDINGS OF THE

AMERICAN MATHEMATICAL SOCIETY

Volume 126, Number 5, May 1998, Pages 1345-1348

S 0002-9939(98)04210-5

\title{
A RELATION BETWEEN HOCHSCHILD HOMOLOGY AND COHOMOLOGY FOR GORENSTEIN RINGS
}

\author{
MICHEL VAN DEN BERGH
}

(Communicated by Lance W. Small)

\begin{abstract}
Let " $H H$ " stand for Hochschild (co)homology. In this note we show that for many rings $A$ there exists $d \in \mathbb{N}$ such that for an arbitrary $A$ bimodule $N$ we have $H H^{i}(N)=H H_{d-i}(N)$. Such a result may be viewed as an analog of Poincaré duality.

Combining this equality with a computation of Soergel allows one to compute the Hochschild homology of a regular minimal primitive quotient of an enveloping algebra of a semisimple Lie algebra, answering a question of Polo.
\end{abstract}

In the sequel the base field will be denoted by $k$. Let $\mathfrak{g}$ be a semisimple Lie algebra and let $A$ be a regular minimal primitive quotient of $U(\mathfrak{g})$. The Hochschild cohomology of $A$ was computed by Soergel in [6] and shown to be equal to the cohomology of the corresponding flag variety. Soergel's computation is rather ingenious and makes use of the Bernstein-Beilinson theorem together with the Riemann-Hilbert correspondence. The case of singular $A$ is still open.

After Soergel's result Patrick Polo asked whether perhaps the Hochschild homology of $A$ also coincided with the homology of the underlying flag manifold. It is indeed rather likely that Soergel's techniques can be adapted to this end.

In this note we answer Polo's question with a different method. Indeed we show, using elementary homological algebra, that for many algebras $A$ (in particular those considered above) we actually have

$$
H H^{i}(N)=H H_{d-i}(N)
$$

for some $d \in \mathbb{N}$ and for an arbitrary $A$-bimodule $N$ (see Corollary 6 ). This can be considered a kind of Poincaré duality. In more general situations (1) may remain true provided we twist $N$ on the right hand side by an automorphism or an invertible bimodule (see Theorem 1).

(1) may be used to complete the results in [7] where the Hochschild homology of a generic three dimensional type A regular algebra was computed (see [1] for terminology). Since we show below that (1) holds for such a algebras, we now also obtain the Hochschild cohomology without any extra work.

Our basic result is the following theorem, which is easily proved.

Theorem 1. Assume that $A$ is an algebra such that there exists a $d \in \mathbb{N}$ with the property that $\operatorname{Ext}_{A^{e}}^{i}\left(A, A^{e}\right)=0$ unless $i=d$. Put $U=\operatorname{Ext}_{A}\left(A, A^{e}\right)$, and assume

Received by the editors November 5, 1996.

1991 Mathematics Subject Classification. Primary 16E40.

Key words and phrases. Hochschild homology, Gorenstein rings.

The author is a senior researcher at the NFWO. 
in addition that $U$ is an invertible A-bimodule. Then for every A-bimodule $N$ we have

$$
H H^{i}(N)=H H_{d-i}\left(U \otimes_{A} N\right) .
$$

Proof. To prove this it is convenient to make use of the derived category. We have

$$
\begin{aligned}
H H^{i}(N) & =H^{i}\left(\operatorname{RHom}_{A^{e}}\left(A^{e} A, A^{e} N\right)\right)=H^{i}\left(\operatorname{RHom}_{A^{e}}\left(A^{e} A, A^{e}\right) \stackrel{L}{\otimes_{A^{e}}} N\right) \\
& =H^{i}\left(U[-d] \stackrel{L}{\otimes_{A^{e}}} N\right)=H^{i-d}\left(U \stackrel{L}{\otimes}_{A^{e}} N\right) \\
& =H^{i-d}\left(A \stackrel{L}{\otimes_{A^{e}}}\left(U \stackrel{L}{\otimes_{A}} A\right)\right)=H H_{d-i}\left(U \otimes_{A} N\right) .
\end{aligned}
$$

We now give several situations in which the theorem can be applied. First from [8] we recall some results concerning Koszul algebras.

Proposition 2. Let $A$ be a (graded) Koszul algebra of global dimension d such that $A^{!}$is Frobenius. Then the hypotheses of Theorem 1 are satisfied, and furthermore we have the following precise description of $U$.

Let $\phi^{!}$be the graded automorphism of $A^{!}$such that $\left(A^{!}\right)^{*}=A_{\phi^{\prime}}^{!}$. Furthermore let $\phi$ be the automorphism of $A$ which is adjoint to $\phi^{!}$. Finally let $\epsilon$ be the automorphism of $A$ which is multiplication by $(-)^{m}$ on $A_{m}$. Then

$$
U=A_{\phi^{-1} \epsilon^{d+1}} \text {. }
$$

In particular, (2) specializes to

$$
H H^{i}(N)=H H_{d-i}\left({ }_{\phi \epsilon^{d+1}} N\right) .
$$

The simplest non-trivial Koszul algebras of finite global dimension are those of global dimension three. These have been studied extensively in $[1,2,3]$. Recall that they have a presentation $k\left\langle x_{1}, x_{2}, x_{3}\right\rangle /\left(f_{1}, f_{2}, f_{3}\right)$ in which $x=\left(x_{1}, x_{2}, x_{3}\right)^{t}$, $f=\left(f_{1}, f_{2}, f_{3}\right)^{t}$ can be chosen in such a way that $f=M x, x^{t} M=(Q f)^{t}$ for $3 \times 3$ matrices $M$ and $Q$, respectively with linear an scalar entries. It is shown in [8] that in this case the automorphism $\phi$ is given in terms of the generators by $x \mapsto Q^{t} x$. In particular if $A$ is a type A algebra (see [1]), then (1) holds with $d=3$.

Let us now exhibit another situation in which Theorem 1 applies and which occurs frequently in practice.

Proposition 3. Let $A$ be a k-algebra equipped with a k-linear ascending filtration $\left(F_{n} A\right)_{n \geq 0}$ such that $\operatorname{gr}_{F} A$ is commutative of finite type over $k$. Assume that $\operatorname{gr} A$ is Gorenstein of dimension d. Then the hypotheses of Theorem 1 hold, and furthermore $U$ carries an A-bimodule filtration such that $\operatorname{gr} U$ is equal to $\omega_{\mathrm{gr} A / k}^{-1}$, the inverse of the dualizing module of gr $A$.

Proof. We use the standard spectral sequence

$$
\operatorname{Ext}_{(\operatorname{gr} A)^{e}}^{i}\left(\operatorname{gr} A,(\operatorname{gr} A)^{e}\right) \Rightarrow \operatorname{Ext}_{A^{e}}^{i}\left(A, A^{e}\right),
$$

which converges because we have positive filtrations.

Let $C$ is a commutative Cohen-Macaulay ring of dimension $d$. From the adjunction formula one has

$$
\operatorname{RHom}_{C^{e}}\left(C, \omega_{C^{e}}\right)=\omega_{C}[-d]
$$


Assume now that $C$ is Gorenstein. Then $\omega_{C e}=\omega_{C} \otimes_{k} \omega_{C}$ and $\omega_{C}$ are invertible, and hence

$$
\operatorname{RHom}_{C^{e}}\left(C, \omega_{C^{e}}\right)=\operatorname{RHom}_{C^{e}}\left(C, C^{e}\right) \otimes_{C^{e}} \omega_{C^{e}} .
$$

Combining this yields

$$
\operatorname{RHom}_{C^{e}}\left(C, C^{e}\right)=\omega_{C}[-d] \otimes_{C^{e}} \omega_{C^{e}}^{-1}=\omega_{C}^{-1}[-d] .
$$

Applying this with $C=\operatorname{gr} A$ shows that (3) degenerates, and we obtain

$$
\operatorname{RHom}_{A^{e}}\left(A, A^{e}\right)=U[-d]
$$

where $U$ lives in one degree and where, from a functoriality argument using (3), one obtains that the filtration on $U$ is an $A^{e}$ filtration, hence an $A$ bimodule filtration. Furthermore for this filtration, gr $U=\omega_{C}^{-1}$. It now suffices to apply lemma 4 below to deduce that $U$ is invertible.

Lemma 4. Assume that $A$ is a ring equipped with a positive ascending filtration, and let $U$ be an A-bimodule, equipped with a left limited A-bimodule filtration. If gr $U$ is an invertible gr A-bimodule, then $U$ is an invertible A-bimodule.

Proof. We have to show $[4, \S 1]$ that $U$ is left and right projective and that the canonical maps

$$
\begin{aligned}
& A \rightarrow \operatorname{End}_{A}\left({ }_{A} U\right), \\
& A \rightarrow \operatorname{End}_{A}\left(A_{U}\right)
\end{aligned}
$$

are surjective. The fact that $U$ is projective is standard (see [5, Cor. D.VII.6]). Therefore let us concentrate on (4). (5) is similar. We use the standard spectral sequence

$$
\operatorname{Ext}^{i}\left(\operatorname{gr}\left({ }_{A} U\right), \operatorname{gr}\left({ }_{A} U\right)\right) \Rightarrow \operatorname{Ext}_{A}^{i}(U, U)
$$

which is compatible with (4) and which obviously degenerates. From this we deduce that

$$
\operatorname{gr} \operatorname{End}_{A}\left({ }_{A} U\right)=\operatorname{End}_{A}\left(\operatorname{gr}\left({ }_{A} U\right)\right) .
$$

Combining this with the hypotheses that $\operatorname{gr} U$ is invertible yields that

$$
\operatorname{gr} A \rightarrow \operatorname{gr}_{\operatorname{End}}\left({ }_{A} U\right)
$$

is surjective. Hence (4) is also surjective.

We use the following lemma below.

Lemma 5. Let the notation be as in the previous lemma. Assume that $\operatorname{gr} U$ is left free of rank one and let $\theta$ be the automorphism of $\operatorname{gr} A$ such that $\operatorname{gr} U=(\operatorname{gr} A)_{\theta}$. Then there exists a filtered automorphism $\psi$ of $A$ such that $U=A_{\psi}$ and $\operatorname{gr} \psi=\theta$.

Proof. Let $\bar{u}$ be a generator of gr $U$ such that

$$
\bar{u} y=\theta(y) \bar{u}
$$

and let $u$ be a lifting to $U$. It is easy to see that $u$ is a basis for $U$. Thus there is an automorphism $\psi$ of $A$ such that $u x=\psi(x) u$. If we take the image of this equality in gr $A$, we find that $\bar{u} \bar{x}=\overline{\psi(x)} \bar{u}$. Comparing with (6) yields $\theta(\bar{x})=\overline{\psi(x)}$. From this we easily deduce that $\psi$ is filtered, and gr $\psi=\theta$. 
Corollary 6. Assume that $A$ is equipped with a filtration $\left(F_{n} A\right)_{n \in \mathbb{N}}$ such that $F_{0} A=k$ and such that $\operatorname{gr} A$ is commutative and generated by $\operatorname{gr}_{1} A$. Put $\mathfrak{g}=\operatorname{gr}_{1} A$ with Lie algebra structure deduced from the commutator on A. Assume furthermore that $\operatorname{gr} A$ is Gorenstein and $[\mathfrak{g}, \mathfrak{g}]=\mathfrak{g}$. Then

$$
H H^{i}(N)=H H_{d-i}(N) .
$$

Proof. Since gr $A$ is graded local, we have $\omega_{A}=A$, as $A$-bimodules, and hence we know from lemma 5 that $H^{i}(N)=H_{d-i}\left(\psi^{-1} N\right)$ for some filtered automorphism of $A$ such that $\operatorname{gr} \psi=\operatorname{id}_{\operatorname{gr} A}$. Let us consider $\mathfrak{g}$ as a subspace of $F_{1} A$. For $x, y \in \mathfrak{g}$ we have

$$
y x-x y=[y, x]+\alpha
$$

for some $\alpha \in k$. We also have $\psi(x)=x+\beta(x)$, where $\beta \in \mathfrak{g}^{*}$. Applying $\psi$ to (7) yields

$$
y x-x y=[y, x]+\beta([y, x])+\alpha
$$

and thus $\beta \mid[\mathfrak{g}, \mathfrak{g}]=0$. Hence $\beta=0$, and we are done.

\section{REFERENCES}

1. M. Artin and W. Schelter, Graded algebras of global dimension 3, Adv. in Math. 66 (1987), 171-216. MR 88k:16003

2. M. Artin, J. Tate, and M. van den Bergh, Some algebras associated to automorphisms of elliptic curves, The Grothendieck Festschrift, vol. 1, Birkhäuser, 1990, pp. 33-85. MR 92e:14002

3. __ Modules over regular algebras of dimension 3, Invent. Math. 106 (1991), 335-388. MR 93e: 16055

4. A. Fröhlich, The Picard group of non-commutative rings, Trans. Amer. Math. Soc. 180 (1973), 1-45. MR 47:6751

5. C. Nastacescu and F. Van Oystaeyen, Graded ring theory, North-Holland, 1982. MR 84i:16002

6. W. Soergel, The Hochschild cohomology of regular maximal primitive quotients of enveloping algebras of semisimple Lie algebras, Ann. Sci. École Norm. Sup. (4) 29 (1996), 535-538. MR 97e: 17016

7. M. van den Bergh, Non-commutative homology of some three dimensional quantum spaces, J. K-theory (1994), 213-230. MR 95i:16009

8. __ Existence theorems for dualizing complexes over non-commutative graded and filtered rings, Journal of Algebra, to appear.

Departement Wni, Limburgs Universitair Centrum, Universitaire Campus, Building D, 3590 Diepenbeek, Belgium

E-mail address: vdbergh@luc.ac.be 